\title{
Enhancement of an Auto-Injector Device for Self- Administration of Etanercept in Patients With Rheumatoid Arthritis Confers Emotional and Functional Benefits
}

\author{
Naceur Rekaya · Steven M. Vicik • Bors T. Hulesch (D) • \\ Laura L. McDonald
}

Received: April 7, 2020 / Published online: June 4, 2020

(C) The Author(s) 2020

\begin{abstract}
Introduction: Etanercept is effective in the management of rheumatoid arthritis (RA) and can be self-administered via an auto-injector. While these devices are generally well accepted, some patients are not comfortable with the process of self-administration; this has been cited as a reason for discontinuation of biologic treatment. Alternative routes of administration (e.g., infusion) are more resource intensive. The aim of this analysis was to explore the attributes of auto-injection devices that impact patient confidence and ability to self-administer.
\end{abstract}

Methods: Patients with RA $(n=168)$ and healthcare providers $(n=82)$ in Belgium, Germany, Japan, Spain, and the UK were interviewed $(n=250$ overall). Mock injection procedures were carried out using an auto-injector device with the addition of a sleeve with a

Digital Features To view digital features for this article go to: https://doi.org/10.6084/m9.figshare.12382679.

Electronic Supplementary Material The online version of this article (https://doi.org/10.1007/s40744020-00216-5) contains supplementary material, which is available to authorized users.

N. Rekaya · S. M. Vicik

Pfizer, Walton Oaks, Surrey, UK

B. T. Hulesch $(\varangle) \cdot$ L. L. McDonald

Brains and Cheek, London, UK

e-mail: bors@brainsandcheek.com wider rubber grip. Importance of and performance of the device against a range attributes were captured using a Likert scale (1-7). Disease severity was captured using the Cochin hand function scale.

Results: Device attributes reported by patients to be most important were 'use without assistance' 'ease of administration', 'ease of operation', and 'ease of grip'. The device with additional sleeve performed strongly against these attributes, scoring 6.9 (out of 7), 6.8, 6.8, and 6.6, respectively with no difference observed between countries. Nurses and physicians reported similar responses. Qualitatively, patients reported that stability and grip provided a sense of control and reduced anxiety. Similar overall 'ease of operation' was reported between patients with mild $(n=89)$ or moderate/severe $(n=71)$ disease (score 6.4 vs. 6.5, respectively).

Conclusions: The auto-injector plus sleeve performed strongly against key attributes even in patients with moderate/severe RA and patients with reduced grip strength. The robust grip improved patient confidence and reduced injection-related anxiety. This may be beneficial in patients who are anxious about self-administration, those new to self-administration, and potentially in patients with reduced hand dexterity as a result of either advanced disease or a painful day. 


\section{PLAIN LANGUAGE SUMMARY}

Etanercept is a medicine used to treat rheumatoid arthritis that is given by injection. It can be delivered by patients themselves, in their own homes, using an auto-injection device that looks like a pen. Some patients are not comfortable with the process of self-injecting. Other patients have severe arthritis in their hands that stops them from carrying out self-injection. One auto-injection device (MyClic) has been enhanced by the addition of a sleeve that fits over the top of the pen. This was shown to 168 patients with rheumatoid arthritis, who already use the MyClic 'pen' and 82 doctors and nurses in five countries (Belgium, Germany, Japan, Spain, and the UK). Patients, nurses, and doctors said what they believed to be the most important features of a self-injection device. Generally, patients, nurses, and doctors from the different countries had similar views. Next, the participants scored the modified auto-injection device against those features. The device plus sleeve scored highly, meaning that patients, nurses, and doctors believed it would work well for the patient population tested. Many patients said that with the added sleeve, the self-injection device was more stable and they were able to grip it better. This helped to make patients feel in control and reduced any anxiety or fear they were feeling about their selfinjection. This suggests that the sleeve is a useful addition and may be particularly useful for patients who are nervous about self-injection or have difficulty gripping self-injection devices because of their arthritis.

Keywords: Auto-injector; Ergonomic device; Etanercept; Rheumatoid arthritis

\section{Key Summary Points}

\section{Why carry out this study?}

Many treatments in rheumatoid arthritis are delivered via self-injection, but some patients are not comfortable with this process, or have severe arthritis in their hands that stops them from carrying out the self-injection.

In this study, an auto-injector with an additional sleeve was demonstrated to users of the device and to healthcare professionals in order to explore whether the added sleeve improved the selfinjection experience in any specific patient groups.

We elicited the key features in a selfinjection device, and then asked respondents to score the performance of the device + sleeve solution on these key attributes. We assumed that the sleeve would provide a modified experience, as compared with the auto-injector alone.

\section{What was learned from the study?}

The device plus sleeve scored highly, meaning that patients, nurses, and doctors believed it would work well for the patient population tested. Many patients said that with the added sleeve, the selfinjection device was more stable and they were able to grip it better. This helped to make patients feel in control and reduced any anxiety or fear they were feeling about their self-injection.

This suggests that the sleeve is a useful addition and may be particularly useful for patients who are nervous about selfinjection or have difficulty gripping selfinjection devices because of their arthritis. 


\section{INTRODUCTION}

Etanercept is an anti-tumor necrosis factor (TNF)- $\alpha$ biologic treatment, effective in the management of rheumatoid arthritis (RA) [1, 2], with a safety profile somewhat superior to that of alternative anti- TNF- $\alpha$ treatments [2-4]. Etanercept can be self-administered via autoinjector devices and this has shown to be a preferred method of administering the treatment [4-6] with alternative routes of administration being less convenient [4] and more resource intensive [6]. Self-injection is associated with a wide range of benefits compared to alternative methods, including increased flexibility in the time and place of injection administration, reduced cost to both the patient and healthcare system, reduced travel time, and reduced caregiver burden [6].

While these devices are generally well accepted, self-injection can be associated with a number of challenges. Some patients are less comfortable with the process of self-administration; in a study of 250 patients who had recently discontinued a range of biologic treatments over the previous 12 months, injection experience was cited as the second most common unprompted primary reason to discontinue (18\% of discontinuations, second only to lack of efficacy) and the most common unprompted secondary reason (34\%) [7]. Overall, $41 \%$ patients reported the injection experience as either a primary or secondary reason for discontinuation [7].

Specific challenges associated with injection experience can include: needle phobia, fear and anxiety, concerns about pain, stinging, and other injection site reactions, the fear of incorrectly administering the drug, and the struggle to use a self-injection device while suffering from arthritic pain, and swelling of the hands [8-10]. Many of these factors stem from, or contribute to, patients' lack of confidence, which can negatively influence adherence [11]. However, many of these factors can be addressed or minimized through careful attention to the design of the device [8]. In particular, design features that can increase a patient's confidence in their ability to correctly deliver treatment may have a positive impact [11]. Self-injection device design can help overcome some of these functional and emotional challenges associated with self-injection [10]. It is suggested that the resulting improvements in patient preference, satisfaction, and injection experience may have a positive effect on adherence to therapy [8].

Subgroups of patients who may struggle with auto-inject procedures include those with hand dexterity issues either in association with advanced disease or due to periodic flare-ups [8]. Research into the design of pre-filled syringes has previously shown that ergonomically designed features can help to decrease the forces needed during an injection. This helps overcome some of the challenges stemming from problems with hand dexterity [12].

The focus of this investigation was to explore patient acceptance of an etanercept auto-injector $\left(\mathrm{MyClic}^{\circledR}\right.$, Pfizer) that had been enhanced by the addition of an ergonomic sleeve (MyClic auto-injector with added sleeve), with the purpose of improving patient usability and self-injection experience. The specific objectives of this analysis were to explore which attributes of the enhanced MyClic auto-injector with added sleeve most impacted patient confidence, and their ability to self-inject. A further objective was to identify the principle benefit of the enhanced device and to identify specific patient types that could most benefit from it.

\section{METHODS}

\section{Study Design}

This study consisted of a survey, structured interview, and practical demonstration of an enhanced injection experience using the Enbrel (Etanercept) MyClic auto-injector (Pfizer, Surrey, UK) with a reusable and washable sleeve, (Genia Medical, Inc., Harrisburg, PA, USA) designed to enhance the ergonomics and ease of the self-injection process (Fig. 1). Specifically, the sleeve was designed to increase the width of the pen to make it easier to grip, was composed of non-slip material, and had a lip to allow the hand to rest comfortably during the administration process. The size of the pen was 

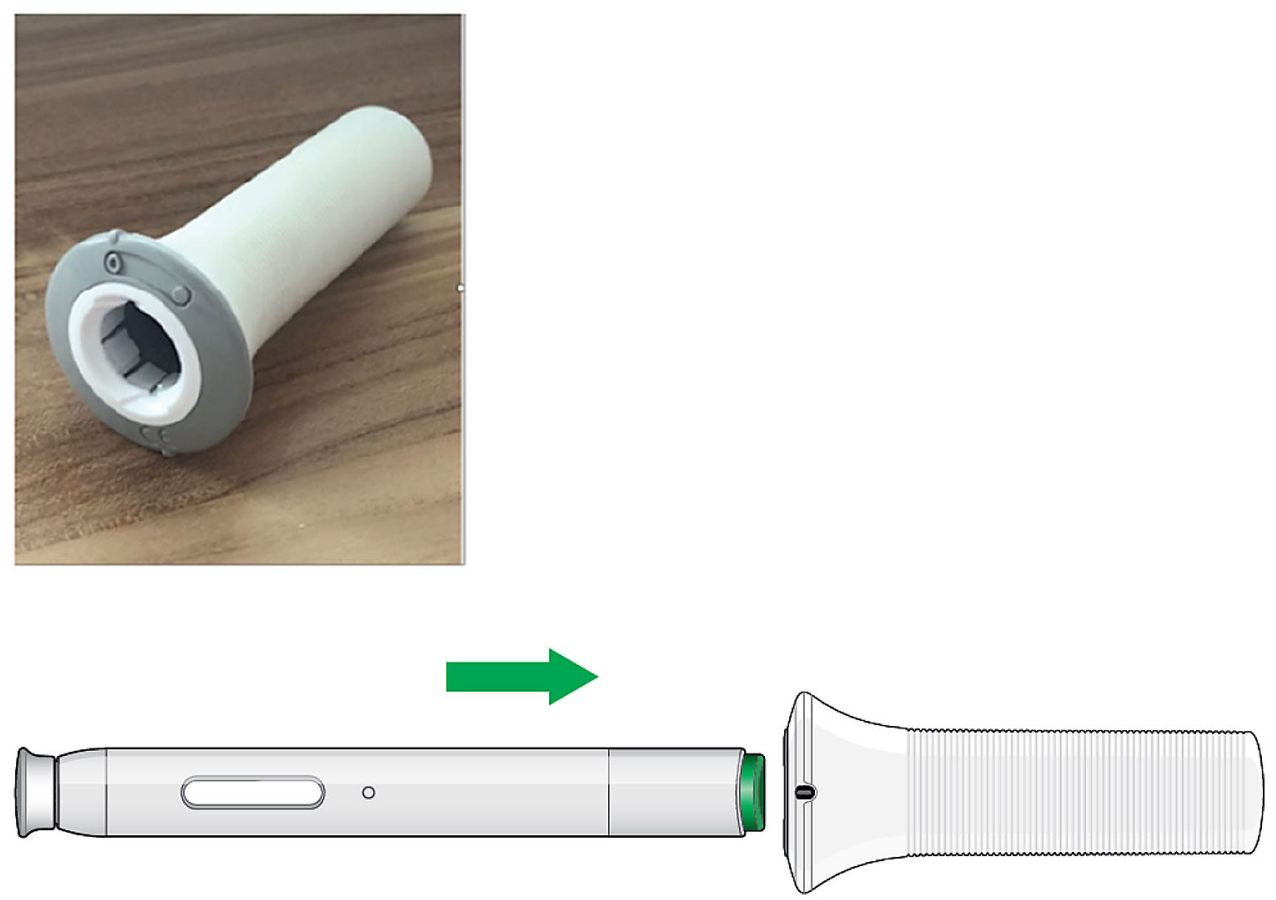

Fig. 1 Auto-inject device with additional sleeve

increased by $59 \%$ by addition of the sleeve. Design of the sleeve ensured that prominent and beneficial features of the auto-injector were not impaired, such as the audible clicks (a signal to confirm successful initiation and completion of the injection) and the viewing window (used by the patient to confirm successful completion of the injection). The MyClic auto-injector is operated by a button located at one end of the pen. This button was purposefully obscured by the end of the sleeve. During the self-injection process, the presence of the sleeve over the auto-injector, automatically triggers the button, meaning that the patient has only to apply pressure to initiate the injection, without the need to physically press the button themselves.

Participants included adult patients with RA. All patients were current MyClic auto-injector users (without the additional sleeve) at the point of enrolment into the study. Participants also included nurses and physicians who were knowledgeable about the care of patients with RA and who had a current caseload of RA patients. The study was conducted in five countries: Belgium, Germany, Japan, Spain, and the UK. Moderators from each individual country were contracted to conduct the surveys in the local language. Moderators were trained and supervised by members of the core team throughout the project.

Research instrument design, participant recruitment, compensation, consent, and data handling were handled according to the guidance provided by the British Healthcare Business Intelligence Association (BHBIA), specifically the Legal and Ethical guidelines of the BHBIA relating to patient research [13]. Participants consented to participate in a demonstration of the product, and they also consented to having their data analyzed for publication in a scholarly journal. Healthcare professionals have also consented to the above. All participants have received compensation for their participation, within the 'fair market value' rules set out by Pfizer.

Mock injection procedures were carried out onto a pad using a demonstration MyClic autoinjector (with no needle and no injectable liquid) with the addition of the sleeve. Participants were shown how to apply the sleeve to the autoinjector and shown how to administer the 
injection onto a pad. Participants were then asked to carry out the procedure themselves.

\section{Data Capture and Analysis}

Disease duration and characteristics were captured. Hand involvement (i.e., the extent to which the patients' hands were impacted by their RA during the day of the survey) was captured using the Cochin Hand Function Scale $[14,15]$. This is a functional disability questionnaire containing 18 questions about a patient's daily activities related to hand function and the degree of difficulty they experience in completing those activities (Supplementary material; Table 1). This tool is presented as a simple-to-use questionnaire, with proven reliability, validity, and responsiveness demonstrated in RA [14, 15]. Questions were scored from 0 (least disability) to 5 (representing greatest disability). The Cochin score for an individual patient was calculated as the sum of the responses for all 18 questions; the scale ranges from 0 to a maximum of 90 where the greater the score the greater the disability. To analyze the impact of hand disability on the response to the enhanced self-inject device, patients were categorized into those with no/ mild hand disability (Cochin score of $0-20$ ) or a moderate/severe degree of hand disability (scores $>21$ ).

Prior to the demonstration of the MyClic auto-injector with added sleeve, all participants were asked how important they felt a range attributes were, in a self-inject device. Importance of these attributes was captured using a Likert scale from 0 to 7 , where 0 indicated that the attribute was "completely unimportant" and 7 indicated that the attribute was "extremely important". Following the demonstration of the MyClic auto-injector with added sleeve, the performance of the MyClic auto-injector with added sleeve was captured; participants were asked how well they felt the autoinjector had performed against each of those same attributes. A similar Likert scale was used from 0 to 7 , where 0 indicated that the device (+sleeve) had "very poor performance" and 7 indicated that the auto-injector had excellent performance. The attributes explored are shown in the Supplementary material, Table 2 , and in Fig. 2. This approach has been used elsewhere $[16,17]$.

Each participant also underwent a structured interview. All responses were captured and qualitative analysis of participant interviews was carried out by comparison of patient responses to each individual question. Emergent themes in response to individual questions were identified and tallies were used to quantify the proportion of respondents expressing these themes.

The study sample size was determined according to feasibility; a minimum of approximately 20 patients from each of the five countries were required to allow sub-analysis. Data analysis was carried out via calculation of mean values. For statistical analysis, Chisquared tests were carried out using SPSS11 and $p$ values of less than 0.05 were considered to be of statistical significance.

\section{RESULTS}

\section{Details of Participants}

Overall, 239 participants were recruited including patients $(n=168)$ who were all current users of the MyClic auto-injector, as well as physicians $(n=43)$ and nurses $(n=39)$ who represented the healthcare professionals caring for patients with RA.

Patient characteristics are shown in Table 1. The 168 patients enrolled were from a range of countries including Belgium $(n=30)$, Germany $(n=41)$, Japan $(n=16)$, Spain $(n=40)$, and the UK $(n=41)$. Patients' average duration of RA was 12.73 years and the average time that patients had been receiving their current medication (etanercept delivered via MyClic autoinjector) was 5.39 years. Three-quarters of patients $(75 \%)$ reported that their RA had impacted their lives to a moderate or significant extent. Also, $55.62 \%$ of patients reported no or mild hand involvement (Cochin scores $\leq 20$ ); the remaining $44.38 \%$ reported moderate or severe degree of hand disability (scores $>20$ ). 


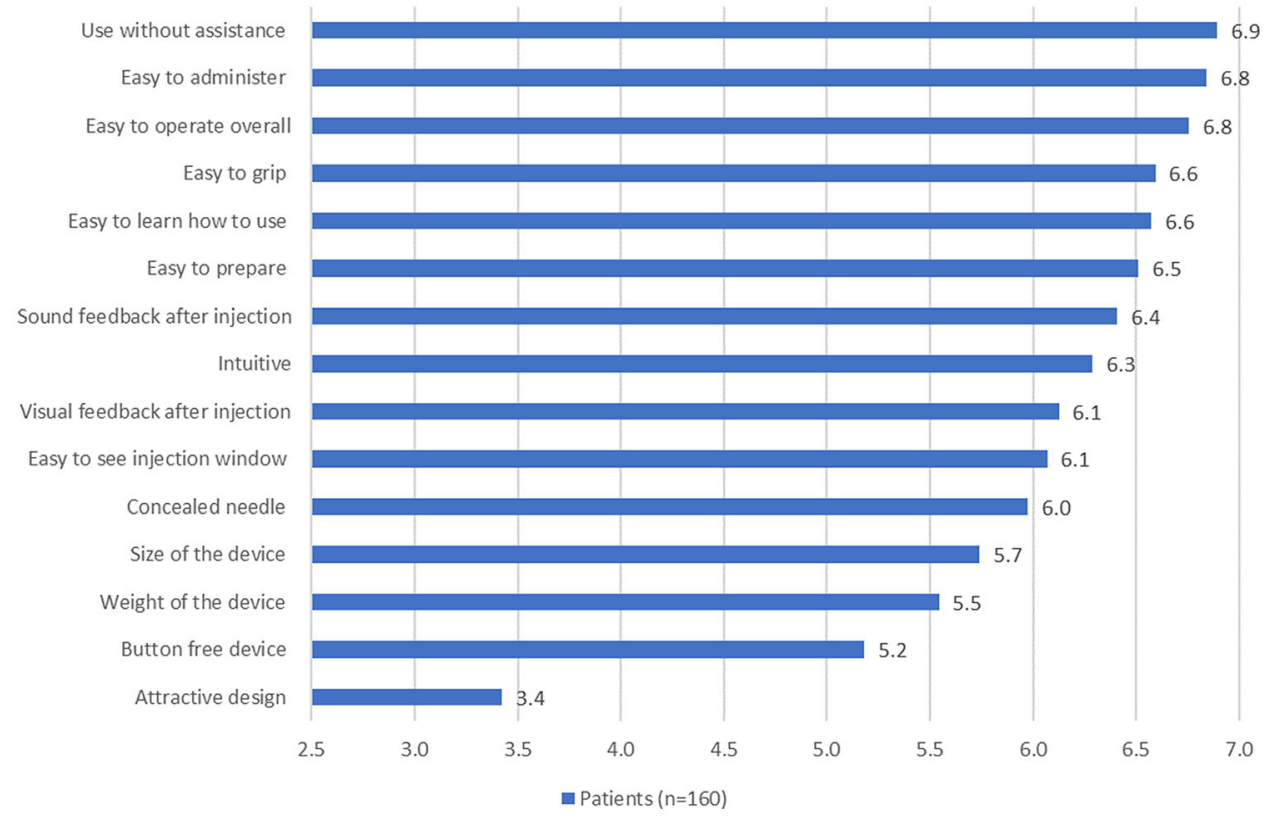

Fig. 2 Importance of device attributes. Participants scored the importance of specific attributes of an auto-injector device using a Likert scale from 1 to 7 , where 1 indicated that the attribute was "not at all important" and 7 indicated that the attribute was extremely important.

Physician and nurse characteristics are shown in Table 2 . The 82 healthcare practitioners enrolled were from a range of countries including Belgium $(n=10)$, Germany, Spain, the UK ( $n=20$ per country), and Japan $(n=12)$. The principle place of work was captured; overall, most physicians and nurses were hospital based (23/43, 53.49\%; 18/39, 46.15\%, respectively) with fewer working in an office or clinic setting, in teaching or university hospitals, or in the community. All nurses were speciality rheumatology nurses. All physicians were specialist rheumatologists except for one orthopedic surgeon (Japan) and one neurologist (Belgium).

\section{Device Attributes}

The overall importance of specific attributes of an auto-injector are shown in Fig. 2 ranked by the perceived importance of each attribute. Attributes considered to be most important by patients were 'use without assistance' (scoring
Mean scores for patients $(n=160)$ are shown. Attributes are ranked in order according to the patient's most important attribute shown at the top of the figure and then in decreasing order of importance

6.9 out of a maximum of 7), 'ease of administration' (6.8), and 'ease of operation overall' (scoring 6.8). Other attributes perceived to be highly important (with average scores over 6 in all groups) included that the device be 'easy to grip' (6.6), 'easy to learn how to use' (6.6), and 'easy to prepare' (6.5) (Fig. 2). Differences in perceived importance of device attributes between patients, nurses, and physicians were explored. There was a very similar pattern of responses between different groups of participants meaning that patients, nurses, and physicians identified similar attributes to be the most important (refer to Fig. 2 for values). Patients and nurses also rated as important (scores $>6$ ) that they received sound feedback after injection (in this case, an audible click) as well as visual feedback after injection, that the injection window was easy to see and that the needle was concealed. For all of these attributes, physicians rated the importance of slightly less important with scores just below 6 (not shown). The attribute perceived to be least important for 
Table 1 Patient characteristics

Country $[n(\%)]$

Belgium

$30(17.86)$

Germany

$41(24.40)$

Japan

$16(9.52)$

Spain

$40(23.81)$

UK

$41(24.40)$

Total

Disease characteristics

Time since diagnosis of RA, mean years (SD)

$12.73(9.67)$

Time on current medication, years (SD)

To what extent has RA impacted patients' life $[n(\%)]^{a}$

No effect

Slight effect

Moderate effect

Significant effect

Cochin score $[n(\%)]$

0 (mild)

$15(9.37)$

$1-20$

$74(46.25)$

$21-40$

$41(25.63)$

$41-60$

$25(15.63)$

$60+($ severe $)$

Total

$160^{\mathrm{a}}(100)$

$R A$ rheumatoid arthritis, $S D$ standard deviation

a Data not provided for a minority of patients

patients was the 'attractive design of the device' (scoring 3.4); nurses and physicians also scored this attribute the lowest (3.8 and 4.0, respectively). In general, physicians tended to give slightly lower scores, but the relative order of importance was similar. Patients and nurses tended to give higher scores and had very similar perceptions of the relative importance of the device attributes; mean scores were more similar between nurses and patients than between physicians and patients.
A separate analysis was undertaken to explore the differences in perceived importance of device attributes between patients in different countries (not shown). With regards to the three attributes identified as most important ('use without assistance', 'ease of administration', and 'ease of operation'), there were no apparent differences between countries; mean scores for each individual country were similarly high for these attributes, all scoring above 6.7 (out of a maximum of 7). Differences between patients from different countries were 
Table 2 HCP characteristics

\begin{tabular}{|c|c|c|c|}
\hline & Nurses $(n=39)$ & Physicians $(n=43)$ & Total $(n=82)$ \\
\hline \multicolumn{4}{|l|}{ Country } \\
\hline Belgium & 5 & 5 & 10 \\
\hline Germany & 10 & 10 & 20 \\
\hline Japan & 6 & 6 & 12 \\
\hline Spain & 11 & 9 & 20 \\
\hline UK & 7 & 13 & 20 \\
\hline \multicolumn{4}{|l|}{ Professional experience } \\
\hline \multicolumn{4}{|l|}{ Principle setting, $n(\%)$} \\
\hline Teaching/university hospital & 8 & 9 & 17 \\
\hline Hospital & 18 & 23 & 41 \\
\hline Community & 2 & 0 & 2 \\
\hline Office/clinic setting & 11 & 11 & 22 \\
\hline
\end{tabular}

All nurses were speciality rheumatology nurses. All physicians were rheumatologists except for one orthopedic surgeon (Japan) and one neurologist (Belgium)

$H C P$ healthcare professional, $R A$ rheumatoid arthritis, $S D$ standard deviation

apparent with some of the other attributes. For example, 'ease of grip', 'intuitive' design of the device, and having a 'button-free device', were less important to Japanese patients (scoring 5.7, 5.5 , and 4.3 , respectively), compared with participants from other countries (scores ranging from 6.4 to $6.9,6.0$ to 6.9 , and 4.8 to 6.0 , respectively). The 'size' and 'weight' and the 'attractive design' of the device were all more important to participants from Belgium and to a lesser extent for respondents from Spain, than from the other countries. Participants from Belgium tended to score consistently more highly than other countries. In general, no other obvious inter-country differences were observed.

After patients, nurses, and physicians had been taken through a demonstration of the enhanced MyClic auto-injector with additional sleeve, they were asked to score how well the enhanced auto-injector performed against those attributes. Patients reported that the auto-injector with additional sleeve performed well against all the attributes (Fig. 3a); in particular, it performed strongly against those attributes ranked as most important including 'use without assistance (scoring 6.8 out of a maximum score of 7), 'ease of administration' (scoring 6.6) and 'ease of operation overall'(scoring 6.5). Nurse and physicians also scored the performance of the enhanced auto-injector highly against the listed attributes. Responses for the top 6 most important attributes are shown in Fig. 3b. No meaningful difference was observed between patients, nurses, or physicians in these attributes.

Importantly, mean scores in all categories and for all categories of participants were greater than 5 , the majority being greater than 6 , on average, indicating a high degree of performance of the auto-injector with added sleeve.

A separate sub-analysis was undertaken to explore how well participants from different countries perceived the auto-injector to perform against the listed attributes and whether any differences could be observed between countries. Similarly high performance ratings were 
a

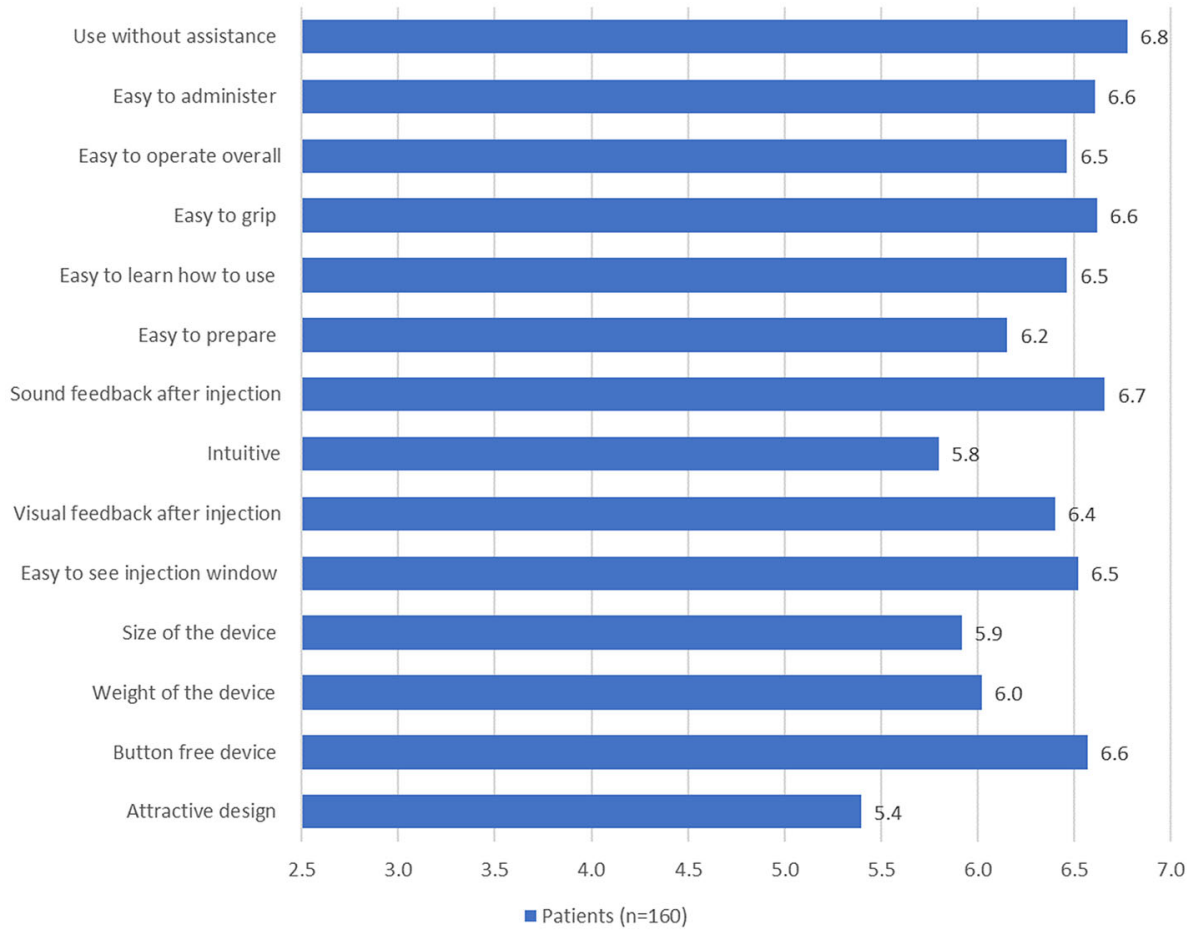

B

Use without assistance

Easy to administer

Easy to operate overall

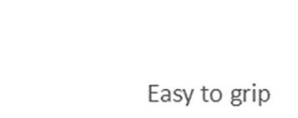

Easy to learn how to use

Easy to prepare

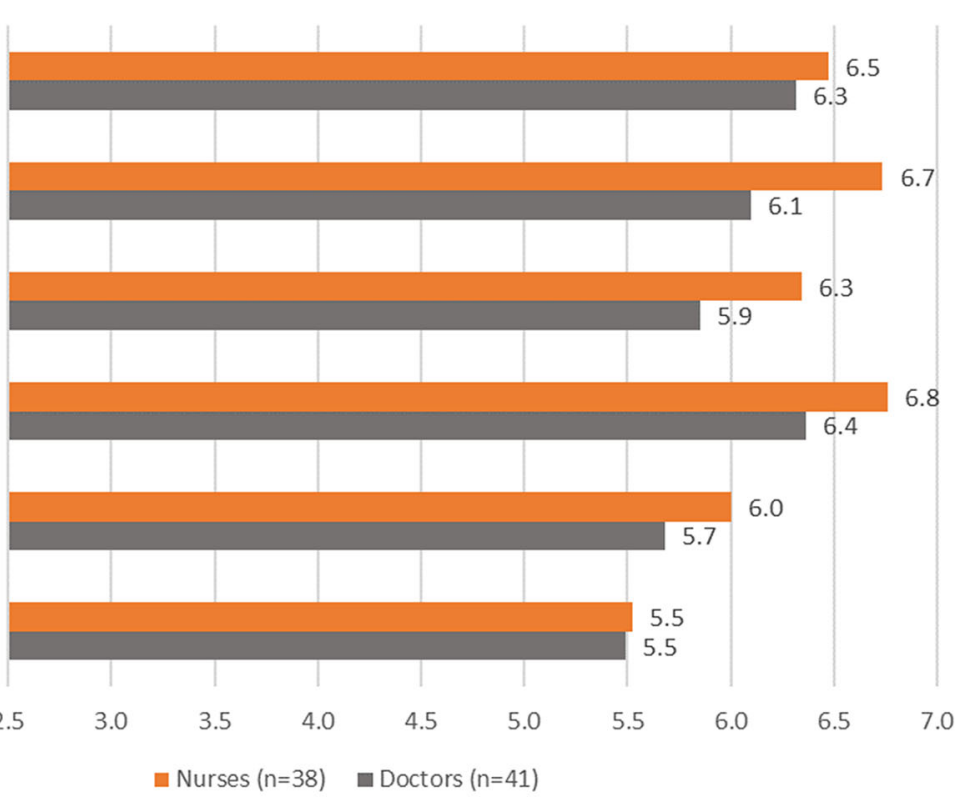

Fig. 3 Performance of the device with the sleeve against key attributes. Participants scored the performance of the auto-injector with the added sleeve, against a variety of attributes using a Likert scale from 1 to 7 , where 1 would indicate that it had "very poor performance" and 7 would indicate that the device had excellent performance. Mean scores for patients $(n=160)$ are shown in a. Responses of physicians $(n=41)$ and nurses $(n=38)$ for the most important attributes are shown in $\mathbf{b}$. Mean patient scores for the most important attributes, divided by country are shown in c. Attributes are ranked in order according to the patient's most important attribute shown at the top of the figure and then in decreasing order of importance 


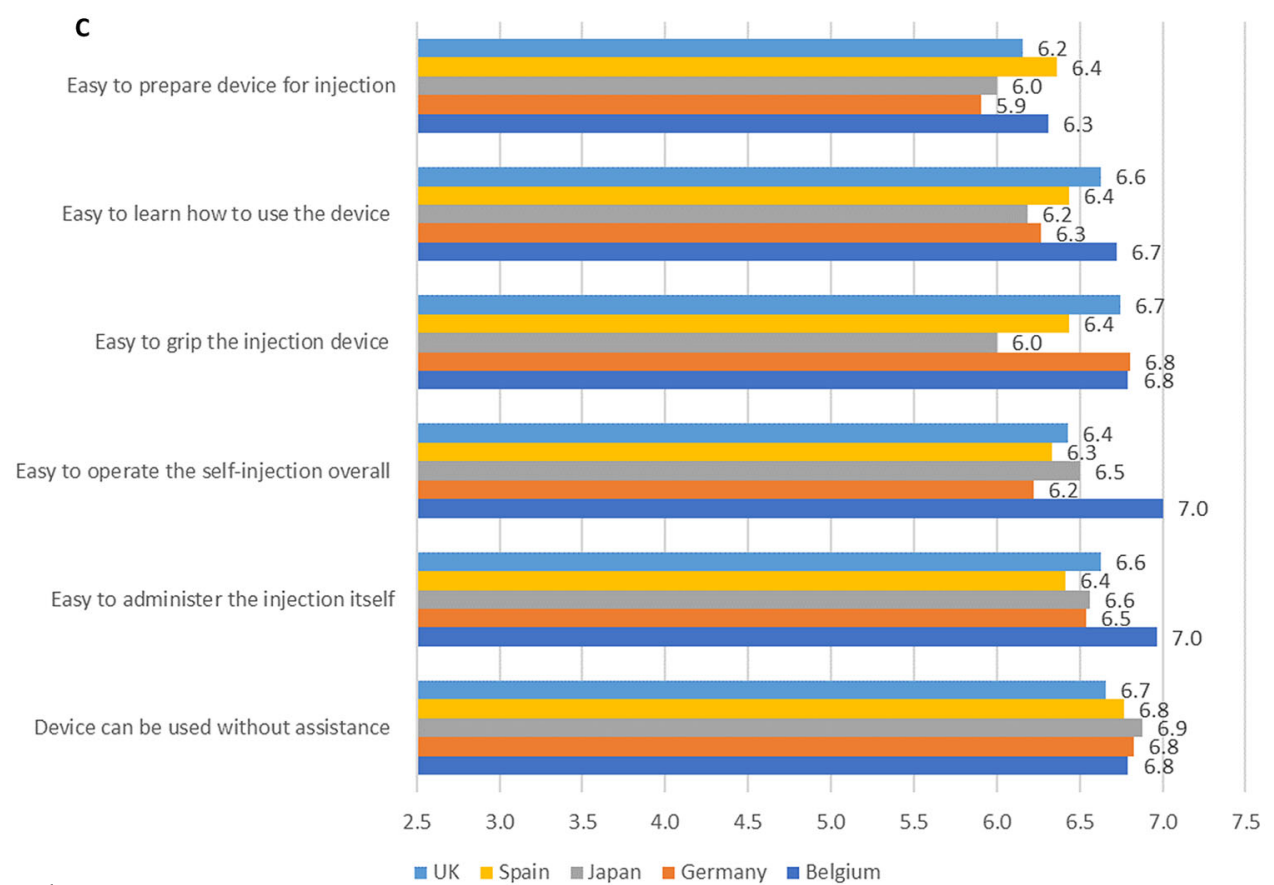

Fig. 3 continued

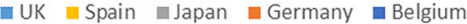

measured for these attributes in all countries notably for 'easy to use without assistance' (mean scores ranging from 6.7 to 6.9/7) and 'easy to administer' (mean scores ranging from 6.4 to 7.0 ; Fig. 3c). For Belgian participants, the auto-injector with additional sleeve scored most strongly for 'easy to administer' and 'easy to operate overall' (both scoring 7/7). Most patients also reported that the device was easy to grip, with mean scores from Belgium, Germany, the UK, Spain, and Japan of 6.8, 6.8, 6.7, 6.4 , and 6.0, respectively). Patients also reported that the auto-injector with additional sleeve scored highly against the attribute 'button free' (mean scores per country ranging from 6.2 to 6.9/7; not shown).

Nurses and physicians were asked how likely they would be to recommend the auto-injector with added sleeve to their patients and to their colleagues on a scale of 1-7 ( $1=$ extremely unlikely $7=$ extremely likely). Nurses and physicians both reported a high likelihood to recommend the auto-injector with added sleeve to their patients (mean scores of 6.6 and 6.2, respectively, Fig. 4). Nurses were more likely to recommend the auto-injector with added sleeve to a colleague compared with physicians ( $6.5 \mathrm{vs.}$ 5.5 , respectively). Patients were extremely likely to recommend the auto-injector with added sleeve to other patients (mean score of 6.3) and highly likely to request it for themselves (5.8, Fig. 4).

\section{Impact of disease severity on patient responses}

A sub-analysis was carried out to compare responses for patients with moderate/severe hand disability (patients with Cochin scores $>$ $21 ; n=71$ ) or no/mild hand disability (Cochin score of $0-20 ; n=89$ ). This was to investigate whether patients with impaired hand dexterity would particularly benefit from the addition of the sleeve. Similar overall 'ease of operation' was reported between patients with no/mild or moderate/severe hand disability (scores of 6.4 vs. 6.5 , respectively, Fig. 5 . This suggested that patients with moderate or severe RA were able 


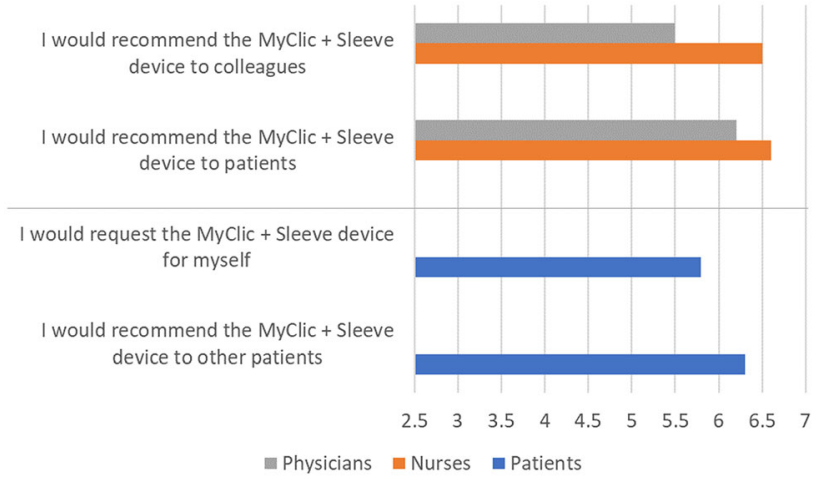

Fig. 4 Likelihood of nurses and physicians to recommend the auto-injector with added sleeve. Nurses, physicians, and patients scored the likelihood of their recommending the auto-injector with the added sleeve using a Likert scale from 1 to 7 , where 1 indicated that they were extremely unlikely and 7 extremely likely to recommend it. Mean scores for patients $(n=160)$, physicians $(n=41)$, and nurses $(n=38)$ are shown

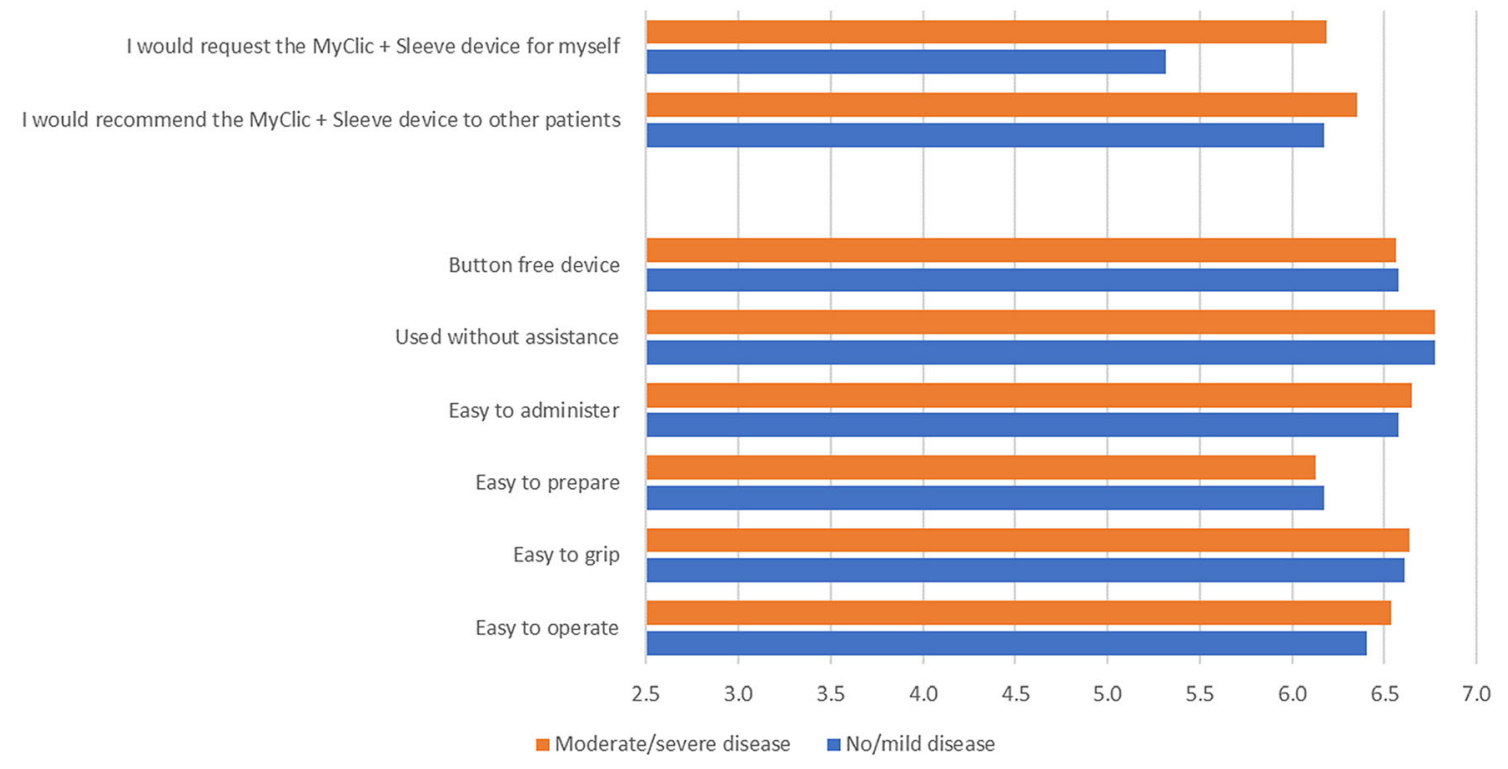

Fig. 5 Effect of disease severity of performance of the auto-injector with added sleeve. Disease severity was measured using the Cochin scale [15]. Participants scored the performance of the auto-injector with the added sleeve against a variety of attributes using a Likert scale from 1 to 7 , where 1 indicated that the device had "very poor

to use the enhanced auto-injector just as easily as patients with less impairment. No difference in other auto-injector attributes were apparent between patients with no/mild or moderate/severe RA including ease of grip, ease of preparation, ease of administration, or ability to performance" and 7 indicated that the device had excellent performance. Mean scores for patients with no/mild hand disability (Cochin score $0-20, n=89$ ), and those with either moderate or severe hand disability (Cochin score 21 or greater, $n=71$ ) are shown

use without assistance (Fig. 5). However, patients with moderate-to-high Cochin scores were significantly more likely to request the sleeve for themselves (6.2 out of a maximum of $7)$ compared with patients with no/mild Cochin scores $(5.3, p<0.05)$, suggesting that patients 
with hand disability as a result of their RA could more easily see the benefit of the additional sleeve.

\section{Themes Arising from Qualitative Interview}

Several recurring themes relating to functional and emotional benefits of the sleeve arose from the qualitative interview.

In terms of functional benefits, patients highlighted that the increased width and nonslip texture provided an enhanced grip on the MyClic auto-injector, resulting in greater confidence to hold and position the device for longer durations. The non-slip texture was also seen as beneficial if an injection was to be performed in moist conditions such as could be experienced in a bathroom. Use of the sleeve allowed displacement of pressure across the whole hand during administration, putting less pressure and stress on the joints, fingers, and wrists. In particular, patients could confidently operate the MyClic auto-injector with the added sleeve without the need to use the thumb, allowing a functional benefit for patients with limited thumb dexterity, or for patients who prefer not to have to press a button to administer the injection. Patients also identified that the sleeve enabled the auto-injector to be held at a $90^{\circ}$ angle without any restriction. These benefits were all recognized to be useful for patients with hand disabilities.

In terms of emotional benefits, patients reported that stability and grip provided a sense of greater control and reduced anxiety. This was equally true for patients with no/mild or moderate/severe hand disabilities. One aspect of the reduced anxiety was related to the button-free format of the auto-injector when the sleeve was in position meaning that patients did not need to separately press the button to initiate the injection, merely apply pressure. A recurring theme was that this offered a release from the anxiety that patients had built up around the action of pressing the button. Patients also reported that the addition of the sleeve allayed their fears that they were not carrying the injection out correctly; in particular, the sleeve was reported to improve stability of placement and reassured patients that the auto-injector was at the correct angle.
Patients reflected that the sleeve may be able to provide greater independence if their condition worsened to such an extent that they would otherwise struggle to self-administer their treatment and that addition of the sleeve may minimize the future need for assistance with administration. Even patients with good hand dexterity on the day of the evaluation recognized the benefit that the sleeve could bring on a 'bad' day when their hands may be stiff and swollen. In some cases, where patients currently required assistance to administer their medication, they felt that the enhancements provided by the sleeve could allow them to selfadminister and not have to rely on a caregiver. Autonomy was found to be an important factor among the patients and one of the preferred benefits of the enhanced MyClic auto-injector with the additional sleeve.

Many patients suggested that the sleeve was something that should be offered to patients new to self-injection, as they felt that it simplified and improved the process; $80 \%$ of patients agreed that they liked or preferred the device with the addition of the sleeve and would accept it if offered to them. A further $15 \%$ of the patient cohort felt that they needed the sleeve to enable them to effectively self-administer their medication.

\section{DISCUSSION}

The addition of a sleeve to the Enbrel MyClic auto-injector was well received by patients, physicians, and nurses. The MyClic auto-injector enhanced by the addition of a sleeve performed very well against the attributes deemed by patients, nurses, and physicians to be most important: 'ease of use without assistance', 'ease of administration', and 'ease of operation overall'. These most important attributes were all related to the use of the device; patient autonomy in administration of medication is of great importance in this population. This was confirmed during the qualitative interviews where the desire to remain autonomous and avoiding the reliance on others for routine tasks such as administration of medication was a common theme. Many patients could see that the sleeve 
could be easier for them to use during future flare-ups when they may otherwise struggle to self-inject he easier grip and increased control provided by the sleeve. Improved autonomy was seen as a major benefit, not only for patients with hand disabilities at the time of the study but also for patients worried about the potential progression of their disease in the future.

We had hypothesized that patients with functional impairment and reduced hand dexterity as a result of severe RA may report the greatest benefit from the addition of the sleeve. Surprisingly, most patients reported benefitting from the sleeve, regardless of their level of hand disability; no difference was observed between patients with moderate-to-severe hand disability (Cochin scores of 21 or above) and those with mild or no hand disability (Cochin scores between 0 and 20). Reassuringly, patients with severe hand disability as a result of their RA were able to confidently use the MyClic autoinjector with additional sleeve, confirming that the sleeve is appropriate for use in this patient subpopulation. A previous study by Hudry et al. [18] explored the impact of hand disability on the ability of patients with RA to self-inject their methotrexate medication. In this study, no difference was found between patients with Cochin scores above or below 20. This is similar to the observation made in this study. This suggests that concerns around correct administration of the medication can often be emotional barriers rather than physical barriers (i.e., inability to carry out the self-injection procedure). Steps taken to address these emotional barriers may therefore have a sizable impact on patient satisfaction and confidence with selfinjection procedures, not only in RA, but potentially in other conditions.

The unforeseen benefits of the additional sleeve, stemming from a number of different areas, were related to the emotional benefits that the sleeve provided. Firstly, the sleeve enhanced the size, grip, and stability of the auto-injector, making patients feel confident in their ability to carry out the injection procedure correctly. Secondly, the sleeve also relieved patient's anxiety that had built up around the self-injection procedure. Previous reports have suggested that diminishing confidence in a patient's own ability to successfully perform a task is linked to their lack of adherence to treatment. As such, increasing a patient's belief in their ability to correctly administer their medication may help increase the level of adherence to that medication [11]. Devices can also impact positively on the self-esteem of elderly patients if they feel they have mastered the device [19]. Addition of the sleeve may therefore have a beneficial effect; the increased confidence and sense of control it gave to patients with resulting impact on confidence in the auto-inject procedure, may boost patient's compliance with treatment. Whether the enhancements described in this study result in improved patient adherence to treatment remains to be evaluated, but is plausible.

These observations suggest that the addition of the sleeve to the auto-inject device may be of greatest benefit to:

- patients who have decreased confidence in their ability to self-inject

- patients with hand impairments due to their RA

- Patients with reduced grip strength

- patients with flaring RA

- patients with any fear or anxiety around the process of self-injection

- patients whose continued autonomy is very important to them

- patients who are new to the process of autoinjection.

Patients also have the choice to perform an injection using the additional sleeve or to continue using the device without the sleeve.

This study has some limitations; we did not explore the impact of patient age on their perception of the additional sleeve or the ability to use it. The study is also a non-comparative study, which does not explore the relative preference of patients of healthcare practitioners to different auto-inject devices. Finally, because a demonstration (sham) device was used, no information relating to the performance of the device in clinical practice could be captured. 


\section{CONCLUSIONS}

Enhancement of current auto-injectors may provide additional functional and emotional benefits for many patients. The MyClic autoinjector, enhanced through addition of an ergonomic sleeve providing a robust grip, performed strongly against key attributes (notably 'ease of administration', 'ease of operation overall', and 'ease of grip') even in patients with moderate/severe RA affecting their hand dexterity. Qualitative analysis of patient interviews clearly identified the importance of not only providing a device that is physically easy to use (functional benefits) but also a device that patients feel confident in using (emotional benefits). The MyClic auto-injector with additional sleeve may be of functional benefit in patients with reduced hand dexterity as a result of either advanced disease or those currently experiencing a disease flare but may be of emotional benefit in a far wider range of patients; the auto-injector with additional sleeve was found to relieve much of the anxiety and increase patient confidence relating to the injection process. This may be particularly beneficial in patients who are already known to be anxious about self-injection. The sleeve may also be of benefit to patients new to self-injection because it enhances the injection experience and may also make administration easier. Patients with decreased confidence in their ability to self-inject, including patients with hand disability as a result of their RA, may also benefit emotionally from using the sleeve, through its potential to increase self-confidence in the process. Future investigations should explore the psychological and emotional impact of auto-injectors as well as the ability of patients to physically use them.

\section{ACKNOWLEDGEMENTS}

Funding. Sponsorship for this study and Rapid Service Fee were funded by Pfizer (Walton Oaks, Surrey, UK). All authors had full access to all of the data in this study and take complete responsibility for the integrity of the data and accuracy of the data analysis.

Editorial Assistance. Editorial assistance in the preparation of this article was provided by Dr. Jenny Smith of JMS Medical Writing Services Ltd and was funded by Pfizer (Walton Oaks, Surrey, UK).

Authorship. All named authors meet the International Committee of Medical Journal Editors (ICMJE) criteria for authorship for this article, take responsibility for the integrity of the work as a whole, and have given their approval for this version to be published.

Disclosures. Naceur Rekaya and Steven M. Vicik are both employees of Pfizer. Bors $\mathrm{T}$. Hulesch and Laura L. McDonald are research directors at Brains \& Cheek (London) LLP, the company that has been commissioned to carry out the research and funded by Pfizer (Walton Oaks).

Compliance with Ethics Guidelines. Research instrument design, participant recruitment, compensation, consent, and data handling were handled according to the guidance provided by the British Healthcare Business Intelligence Association (BHBIA), specifically the Legal and Ethical guidelines of the BHBIA relating to patient research [13]. Participants consented to participate in a demonstration of the product, and they also consented to having their data analyzed for publication in a scholarly journal. Healthcare professionals have also consented to the above. All participants have received compensation for their participation, within the 'fair market value' rules set out by Pfizer.

Data Availability. The datasets generated during and/or analyzed during the current study are not publicly available at the present time but are available from the corresponding author on reasonable request.

Open Access. This article is licensed under a Creative Commons Attribution-NonCommercial 4.0 International License, which permits 
any non-commercial use, sharing, adaptation, distribution and reproduction in any medium or format, as long as you give appropriate credit to the original author(s) and the source, provide a link to the Creative Commons licence, and indicate if changes were made. The images or other third party material in this article are included in the article's Creative Commons licence, unless indicated otherwise in a credit line to the material. If material is not included in the article's Creative Commons licence and your intended use is not permitted by statutory regulation or exceeds the permitted use, you will need to obtain permission directly from the copyright holder. To view a copy of this licence, visit http://creativecommons.org/licenses/bync/4.0/.

\section{REFERENCES}

1. Tarp S, Furst DE, Dossing A, et al. Defining the optimal biological monotherapy in rheumatoid arthritis: a systematic review and meta-analysis of randomised trials. Semin Arthritis Rheum. 2017;46(6):699-708.

2. Singh JA, Hossain A, Tanjong Ghogomu E, Mudano AS, Tugwell P, Wells GA. Biologic or tofacitinib monotherapy for rheumatoid arthritis in people with traditional disease-modifying anti-rheumatic drug (DMARD) failure: a Cochrane Systematic Review and network meta-analysis (NMA). Cochrane Database Syst Rev. 2016;11:Cd012437.

3. Michaud TL, Rho YH, Shamliyan T, Kuntz KM, Choi HK. The comparative safety of tumor necrosis factor inhibitors in rheumatoid arthritis: a meta-analysis update of 44 trials. Am J Med. 2014;127(12): 1208-32.

4. Navarro-Millan I, Herrinton LJ, Chen L, Harrold L, Liu L, Curtis JR. Comparative effectiveness of etanercept and adalimumab in patient reported outcomes and injection-related tolerability. PLoS One. 2016;11(3):e0149781.

5. Borras-Blasco J, Gracia-Perez A, Castera MD-E, Rosique-Robles J, Abad J. Educational session as a tool to increase patient satisfaction of switching etanercept from the prefilled syringe to the autoinjection pen. Expert Opin Biol Ther. 2013;13(8):1103-8.

6. Borras-Blasco J, Gracia-Perez A, Rosique-Robles JD, Castera MD, Abad FJ. Acceptability of switching adalimumab from a prefilled syringe to an autoinjection pen. Expert Opin Biol Ther. 2010;10(3): 301-7.

7. Bolge SC, Goren A, Tandon N. Reasons for discontinuation of subcutaneous biologic therapy in the treatment of rheumatoid arthritis: a patient perspective. Patient Prefer Adher. 2015;9:121-31.

8. van den Bemt BJF, Gettings L, Domanska B, Bruggraber R, Mountian I, Kristensen LE. A portfolio of biologic self-injection devices in rheumatology: how patient involvement in device design can improve treatment experience. Drug Deliv. 2019;26(1):384-92.

9. Schiff M, Saunderson S, Mountian I, Hartley P. Chronic disease and self-injection: ethnographic investigations into the patient experience during treatment. Rheumatol Ther. 2017;4(2):445-63.

10. Keininger D, Coteur G. Assessment of self-injection experience in patients with rheumatoid arthritis: psychometric validation of the Self-Injection Assessment Questionnaire (SIAQ). Health Qual Life Outcomes. 2011;9:2.

11. Nafradi L, Nakamoto $\mathrm{K}$, Schulz PJ. Is patient empowerment the key to promote adherence? A systematic review of the relationship between selfefficacy, health locus of control and medication adherence. PLoS One. 2017;12(10):e0186458.

12. Sheikhzadeh A, Yoon J, Formosa D, Domanska B, Morgan D, Schiff $M$. The effect of a new syringe design on the ability of rheumatoid arthritis patients to inject a biological medication. Appl Ergonom. 2012;43(2):368-75.

13. BHBIA guidelines. https://www.bhbia.org.uk/ guidelines-and-legislation/legal-and-ethical-guideli nes. Accessed 8 July 2019.

14. Poiraudeau S, Lefevre-Colau MM, Fermanian J, Revel M. The ability of the Cochin rheumatoid arthritis hand functional scale to detect change during the course of disease. Arthritis Care Res. 2000;13(5):296-303.

15. Duruoz M, Poiraudeau S. Development and validation of a rheumatoid hand functional disability scale that assesses functional handicap. J Rheumatol. 1996;23(7):1167-72.

16. Thakur K, Biberger A, Handrich A, Rezk MF. Patient perceptions and preferences of two etanercept autoinjectors for rheumatoid arthritis: findings from a patient survey in Europe. Rheumatol Ther. 2016;3(2):245-56.

17. Tischer B, Mehl A. Patients' and nurses' preferences for auto-injectors for rheumatoid arthritis: results of 
a European survey. Patient Prefer Adher. 2018;12: 1413-24.

18. Hudry C, Lebrun A, Moura B, Zinovieva E, Backers $\mathrm{O}$, Herman-Demars H. Evaluation of usability and acceptance of a new auto-injector intended for methotrexate subcutaneous self-administration in the management of rheumatoid arthritis. Rheumatol Ther. 2017;4(1):183-94.

19. Thomson R, Martin JL, Sharples S. The psychosocial impact of home use medical devices on the lives of older people: a qualitative study. BMC health Serv Res. 2013;13:467. 